\title{
A review on wearable inertial tracking based human gait analysis and control strategies of lower-limb exoskeletons
}

\begin{abstract}
In recent years, human motion tracking based on inertial sensors took a huge leap forward as a cost effective enabling technology. It overcomes the limitations of existing tracking systems such as magnetic, mechanical and optical in terms of power, cost and size. It has high impact in the areas of human performance assessment and human-robot interaction as illustrated in gait analysis and assistive devices control. It can be used as a method for alternate gait analysis overcoming the limitations of motion capture systems which are restricted to indoor environment. Also, human motion intention estimation is made possible with the help of these devices and is useful in exoskeleton control. This paper surveys the role of inertial based systems in human gait analysis and lower limb exoskeleton control. In the later part of the paper, state-of-the-art control strategies designed for lower limb exoskeletons are reviewed that show the application of human motion sensing.
\end{abstract}

Keywords: human robot,human motion, magnetic, mechanical
Volume 3 Issue 7 - 2017

\author{
Prudhvi Tej Chinmilli, Sangram Redkar, \\ Wenlong Zhang, Tom Sugar \\ The Polytechnic School,Arizona State University, USA
}

Correspondence: Sangram Redkar, The Polytechnic School, Arizona State University, USA, Email sredkar@asu.edu

Received: September 06, 2017 | Published: December 28, 2017

\section{Introduction}

Human gait analysis has become an active area of research from the past few decades due to its applications in the fields of medicine, sports and identification of people for security reasons. It gained a huge popularity in medical field as it provides key information about person's quality of life. It gives reliable information about the evolution of different diseases such as stroke, Parkinson's and cardiopathies. The effects due to ageing in elderly people can be monitored through gait analysis. The technologies used for gait analysis can be classified as wearable or non-wearable sensors. Nonwearable sensors require the use of controlled research facilities where movements are captured by camera based analysis systems and ground contact forces are recorded by floor sensors. Camera based systems can provide three-dimensional position of the body segments. The limitations of these non-wearable sensors entail high setup cost and are restricted to laboratory environment. Also, it is difficult for the subjects to visit the lab more frequently. This provides motivation to develop sensors which are of low cost, portable and not restricted to the indoors. Recent advancements in MEMS technology paved a way to develop these types of sensors.

The questions related to quality care in areas with reduced access and how to maximize the participation of individuals with disabilities can be answered by wearable technology. Remote monitoring has the potential to mitigate problematic patient issues that is possible with the aid of wearable sensors. Also, these wearable sensors are useful in rehabilitation procedure for the patients who capture vital signs in their movements and provide feedback to the physicians. Another important application of inertial based tracking is in the control of assistive devices. It provides the kinematic configuration of the segment for a given wearable exoskeleton. Also, it is desirable to configure a non-cumbersome solution that allows free movement regardless of the context of application. A wearable motion capture system can be built of small inertial measurement units (IMUs) mounted on the exoskeleton structure. This paper reviews the four important aspects:

a. Constraints of human body segments and fixation methods.

b. Comparison of different motion captures trackers.

c. Human gait analysis using wearable sensors.

d. Control strategies of lower-limb exoskeleton.

The paper is organized as follows: Second section describes the kinematics of human biomechanics. The comparison between different motion capture trackers is given in third section. Fourth section details human gait analysis using wearable sensors. The stateof-the-art control strategies in lower-limb exoskeletons are reviewed in fifth section. Finally, conclusion is given in sixth section.

\section{Human body kinematics}

\section{Importance of exploring kinematic constraints of human body segments}

In many studies, incorporating biomechanical model into tracking system showed significant reduction in estimation errors. Human body can be represented as system of rigid segments connected in chains. To reduce the drift in sensors, most of the studies included physical constraints to confine the estimates within the range of motion. In a recent study, researchers developed an algorithm to estimate uniaxial joint angles based on the measurements from the sensors mounted on thigh and the shank. ${ }^{1}$ Here, they incorporated lower limb constraints in the system and results showed a significant decrease in estimation errors. This algorithm was validated in the subject performing various gait phases. In a further study by Favre et al., ${ }^{2}$ authors corrected knee 
joint angles by including joint constraints using inclination information from accelerometer during static periods. ${ }^{2}$ In order to measure forearm orientation relative to the upper arm, they implemented physical constraints of the elbow. Error minimization was done using the knowledge that elbow joints do not perform abduction/adduction. ${ }^{3}$ In a study to estimate knee joint angles using 2 IMUs mounted on the thigh and the shank. Researchers introduced biomechanical constraints into the tracking system. ${ }^{4}$ Performance of the algorithm was evaluated for different tasks of walking and running. There was an average error of $0.7 \mathrm{deg}$ for slow walking to $3.4 \mathrm{deg}$ for running for a simplified knee model.

In order to describe joint configuration, Denavit Hartenberg convention is typically used to define orientation and position of one link with respect to another link. Human body segments orientation is different from robot configuration. Human body configuration lacks even surfaces and right angles. In most of the studies, the assumption that the local coordinate axis of the sensor aligns with the joint axis was made. For an instance, for a lower limb biomechanical model of lower limb incorporated into a tracking system, the lower limb of human skeleton comprised of three primary joints namely hip, knee and ankle. ${ }^{5}$ According to a comparative study, minimum Degrees of Freedom (DOF) possible for hip, knee and ankle is one (flexion/extension). Based on the problem statement, researchers modeled human limb with different number of DOF for each joint. For an example, if a person wanted to turn in both directions hip can be modeled using 2 DOF (Flexion/Extension \& Internal/External rotation), knee, ankle can be modeled using 1DOF. For most of the tasks, lower limb exoskeleton can be modeled using a total number of 7 DOF in a leg considering 3 DOF of the hip joint, 1 DOF of the knee and $3 \mathrm{DOF}$ of the ankle. It was described that hip abduction/adduction and internal rotation do not play a key role in the walking cycle. ${ }^{6}$ It is very important to consider the range of motion of different joints for modeling of body segments. In general, Table 1 lists out the range of motion of lower limb joints. ${ }^{7}$ Taking number of degrees of freedom of the joints in to consideration, the joints are modeled as mechanical joints in order to develop a kinematic model. Therefore, Kinematic constraints are exploited to obtain the position and orientation vector of the joints in the local coordinates of the sensors.

Table I illustrates comparison between non-wearable system and wearable systems, merits and drawbacks of both the systems

\begin{tabular}{lllll}
\hline Joint & Motion & $\begin{array}{l}\text { Human } \\
\text { Maximum }\end{array}$ & $\begin{array}{l}\text { Walking } \\
\text { Maximum }\end{array}$ & $\begin{array}{l}\text { Operational } \\
\text { Limits }\end{array}$ \\
\hline Hip & Flexion & $120 \mathrm{deg}$ & $32.2 \mathrm{deg}$ & $90 \mathrm{deg}$ \\
& Extension & - & $22.5 \mathrm{deg}$ & $25 \mathrm{deg}$ \\
Knee & Flexion & $120 \mathrm{deg}$ & $73.5 \mathrm{deg}$ & $90 \mathrm{deg}$ \\
Ankle & Flexion & $50 \mathrm{deg}$ & $14.1 \mathrm{deg}$ & $20 \mathrm{deg}$ \\
& Extension & $20 \mathrm{deg}$ & $20.6 \mathrm{deg}$ & $15 \mathrm{deg}$ \\
\hline
\end{tabular}

\section{Fixation methods}

Sensors position and orientation mounted on segments of the body are characterized by joint axis and positions, respectively. A good fixation method reduces artifacts due to skin movement. Most of the papers ascertain that the good fixation method can significantly reduce the errors caused by improper alignments of the joint axis. Double side adhesive tape, ${ }^{8}$ elastic straps, ${ }^{9,10}$ Velcro straps ${ }^{11}$ and neoprene straps $^{12}$ are commonly used on subject's bodies. However, errors caused by skin movement are significant. Sensor enclosure can reduce the movement of the skin and avoid sensors from damage. Semi rigid belt, ${ }^{13}$ exoskeleton harness ${ }^{14}$ are used for better sensor attachment which restrict subjects from normal movement.

\section{Calibration motion and postures}

The main objective of the fixation methods is to ensure that the local coordinate axis of the sensor is aligned with the anatomical joint axis. In some papers, authors ${ }^{15,16}$ made the subject to stand vertical for few seconds and used the acceleration measured during that time interval to determine the local coordinates of the joint axis. Some used sitting calibration postures. ${ }^{16}$ Predefined calibration motions can also be used instead of static postures to identify the sensors to segment orientations. ${ }^{14,17} \mathrm{~A}$ combination of postures and motions can be used to identify orientations. Here in this protocol, flexion/extension motions were used along with the static postures to identify local coordinates of the joint axis. ${ }^{18}$ This protocol was used to solve the kinematic chain to refine joint axis orientation and position from the combination of calibration postures and motions and manual measurement of dimensions of segment. Some researchers introduced methods to overcome the limitations of identifying joint axis by predefined calibration postures and motions by exploring kinematic constraints. ${ }^{19}$

\section{Discussion}

The primary problem for the IMU based human gait analysis is that the local coordinate axis of the sensor is not aligned with the joint axis. This problem is ignored in most of the papers. Most of the papers tried to address this problem using predefined calibrated motion or postures to identify joint axis coordinates in the local sensor. This led to many errors as this process depends on the precision with which performs predefined postures and movements. Some possible improvements to existing approaches may include:

a. Modeling body segments exploring flexible constraints by introducing some compliance in to the model to accurately capture kinematics of the human body.

b. Relaxing assumptions related to mounting sensor on segments

c. Measurement technique should not rely on any predefined calibrated postures or movements and should be able to find joint coordinates in any arbitrary position or orientation.

\section{Human motion capture technologies}

The need for accurate human motion tracking depends on requirements like accuracy, update rate, fidelity, bandwidth and intended application of devices and algorithms. Human motion tracking can be achieved using wide list of sensors and technologies which include mechanical, magnetic, optical, acoustic and inertial tracking. Kinematic models along with the observed data from the sensors using tracking algorithms can be used to estimate the movements. Various technologies, their advantages and limitations are discussed in this section.

\section{Mechanical trackers}

This is one of the oldest tracking technologies. These systems are fairly accurate and have low latency. Mechanical trackers are divided in to two categories: 
a. Body-based

b. Ground-based

Body based systems generally involves body based linkages and Goniometers. These angle measuring devices provide joint angles in the kinematic algorithms which determine the position of end effectors and body posture. Body based systems need to be recalibrated for every user due to difference in anthropometric measurements. This recalibration is complicated and time consuming. These systems are not flexible; users cannot interact to physical objects in environment in a natural way. Once these systems are designed, they cannot be modified very easily. These entire limitations make these body based mechanical trackers difficult to use. Ground based mechanical tracker system has six degrees of freedom. These typically contains boom which is either grasped by user or attached to a device worn by user. Encoders give body posture measurement with respect to synthetic environment. These ground based tracking devices have a limited range of operation. Mechanical trackers are fairly accurate and inexpensive. But due to several limitations, they do not provide accurate tracking in a large working space. Body based systems are difficult to calibrate and are cumbersome. On the other hand, ground based mechanical trackers have limited range of operation and are suitable to track single subject in limited range.

\section{Magnetic trackers}

Magnetic trackers determine position and orientation using small sensors mounted on the body to sense the generated magnetic fields. These sensors contain three mutually orthogonal coils.. ${ }^{20}$ The induced currents in each of the three sensor coils used to calculate position and orientation. These magnetic trackers accuracy depends on the characteristics of local magnetic field. Magnetic tracking systems are susceptible to interferences which makes them unsuitable for robust tracking applications. The presence of any magnetic materials in the vicinity can affect the performance of these. ${ }^{21}$ The more critical drawback of these devices is a very limited range of operation. These limitations restrict magnetic tracking to operate in a small working volume.

\section{Optical trackers}

The optical systems use a wide range of technologies. The cost and performance of different optical systems differ widely. The active or passive markers on the body can capture motion. Optical tracking can be divided in to three categories

a. Pattern recognition systems: these systems can sense artificial pattern of lights to determine position and orientation.

b. Image Based Systems: these systems multiple cameras to track predetermined points on moving objects within the working space. $^{22}$

c. Structured Light and Laser Systems: These systems use laser or beamed light to create a plane of light that is swept across the image. Some systems use a laser to scan points, the entire scene systematically or randomly to determine positions.

The major drawbacks of optical trackers are related to cost and restricted use in a structured environment like laboratories. They are very susceptible to skin movements. The primary drawback of pattern recognition systems is their inability to track in all orientations.
Optical tracking using image based systems requires costly cameras to accurately track human movement. They largely depend on Computer vision techniques which are computationally very demanding and expensive to capture high resolution motion in real time. ${ }^{23}$

\section{Acoustic trackers}

Acoustic trackers are inexpensive alternative to magnetic trackers. They have more range and not susceptible to interferences. They use emitters and receivers of sound waves. Acoustic trackers calculate position based on the speed of sound and upon the wavelength of an acoustic signal. These systems are adversely affected due to echoes and reflections of sound waves. They are very sensitive to factors like changes in temperature, wind flow etc.

\section{Inertial measurement systems}

Recent developments in MEMS technology have made inertial sensors cost effective, miniature and easily available. These advances have been driven by rapidly developing market for low cost automotive vehicle navigation and control systems. Inertial measurement systems easily overcome the limitations of other motion capture technologies. They can be easily strapped to any human body segment and can be used anywhere. Unlike, other technologies inertial sensing is not associated with inherent latency. They can be used practically and can offer low cost systems for data acquisition. Inertial sensing can also be augmented to other motion capture technologies. There are certain limitations associated with inertial tracking but can easily be overcome by rapid advancements in tracking algorithms.

\section{Comparison between non-wearable and wearable exoskeleton}

In general, motion capture systems can be divided into nonwearable and wearable systems. Non-wearable systems are very costly and restricted to laboratory environments. But, recent developments devices like 'Kinect' make it easy to conduct validation experiments. In contrast to non-wearable system, the miniaturized sensors have made it possible to carry the system anywhere and different aspects of the gait can be processed in real time. Sensors such as accelerometer, gyroscopes, and pressure sensors and so on may provide low cost wearable gait analysis system. Table 1 illustrates comparison between non-wearable system and wearable systems, merits and drawbacks of both the systems.

\section{Human gait analysis using wearable sensors}

Researchers have focused on developing alternate gait analysis method based on wearable sensors to overcome the limitations of conventional motion capture systems and have shown great progress in the last two decades. Using Wearable sensors like accelerometers, gyro sensors, inclinometers, Goniometers, force sensors and so on. The need to develop low cost, portable devices for measuring motion has driven researchers to develop wearable system using these sensors. However, there are issues related to the accuracy of the measured data from these sensors. Many research papers discuss the issues and have presented different methodologies to reduce the errors and improve accuracy. In this section, a brief review on basics and parameters of interest of human gait, use of wearable sensors in gait analysis, instrumentation used in various experimentation, gait analysis method configuration based on wearable sensors, importance 
of inertial sensors in gait analysis, various inertial sensor algorithms, applications of wearable sensors in gait analysis are presented.

\section{Basics and parameters of interest for human gait}

Gait phase: Human walk can be considered as the periodic movement of the body segments. In order to analyze walking, separated gait phases are observed and studied. In the past, normal events were conventionally used as the critical actions of separated gait phases. This practice failed in the case of impaired patients as their style is different from normal walking. ${ }^{24}$ Gait phases give critical information related to different movements generated at individual joints and segments during walking as shown in Figure 1. In general human walking gait cycle is divided in to eight phases that is initial contact, loading response, mid stance, terminal stance, pre-swing, initial swing, mid swing and terminal swing. Table 2 gives gait sub phases along with their interval in a total gait cycle.

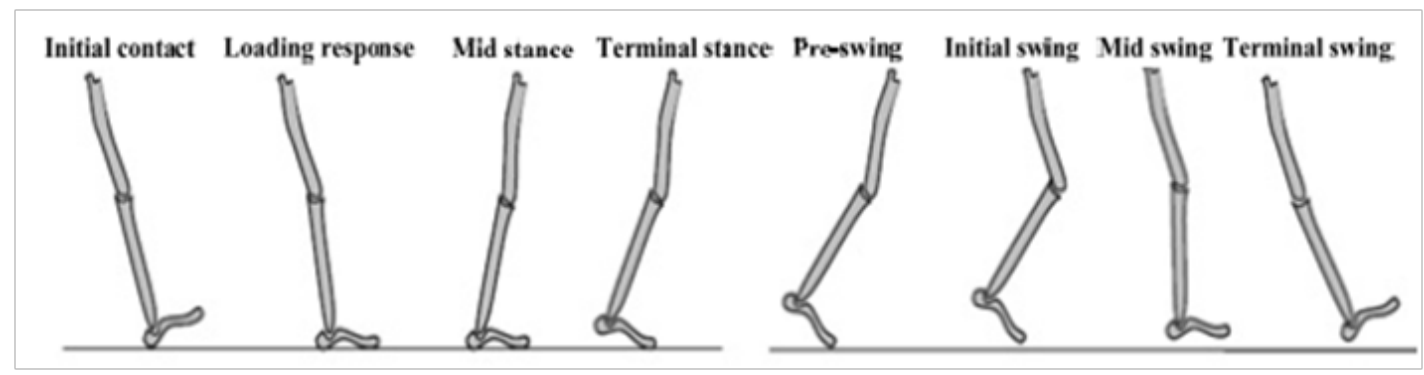

Figure 1 Gait sub phases. ${ }^{25}$

Table 2 Gives gait sub phases along with their interval in a total gait cycle

\begin{tabular}{lll}
\hline System & Merits & Limitations \\
\hline $\begin{array}{l}\text { Non-wearable } \\
\text { system }\end{array}$ & $\begin{array}{l}\text { Thexibility in controlling measurements in real time } \\
\text { Allows more precision, better repeatability, reproducibility and less } \\
\text { interference from external environment }\end{array}$ & $\begin{array}{l}\text { Measurement analysis is restricted to } \\
\text { laboratory space. } \\
\text { Expensive equipment's and } \\
\text { experimentation }\end{array}$ \\
& $\begin{array}{l}\text { Allows simultaneous analysis of multiple gait parameters } \\
\text { Limited range of operation }\end{array}$ \\
& $\begin{array}{l}\text { Portable and low cost systems } \\
\text { Transparent analysis and gait activities can be monitored for the long period } \\
\text { of time }\end{array}$ & $\begin{array}{l}\text { Allows evaluation of limited number of } \\
\text { gait parameters. }\end{array}$ \\
system & Increased availability of miniaturized sensors & Susceptible to noise and \\
& Allows the integration of sensors in any place & Interference of external factors.
\end{tabular}

\section{Human gait's parameters of interest}

Analysis of human gait has its applications in various fields. Parameters of interest vary for different tasks. For instance, in the field of sport, an athlete's performance is assessed by evaluating muscle contraction force through EMG. ${ }^{26}$ For people with Parkinson's disease and stroke, Salarin et al. ${ }^{27}$ performed gait measurements and concluded that the stride length is highly correlated with the severity of the disease. ${ }^{27}$ In the clinical field, human gait analysis becomes important in evaluating gait disorders, neurodegenerative diseases such as multiple sclerosis, cerebellar ataxia, brain tumors and so on. Multiple sclerosis patients show alterations in step size, walking speed. ${ }^{28}$ Another condition related to gait disorders is osteoporosis, which is commonly seen in elderly people. ${ }^{29}$ Evaluation of gait parameters is helpful for diagnosis in early stages. Accurate analysis of human gait is only possible with evaluation of gait parameters. Below is the table which gives an overview of gait parameters regularly used for research in clinical, sports and recognition field. ${ }^{30}$ (Table 3) Accurate evaluation of gait parameters plays a key role in improving skills, abilities for sportspersons. In medical field, a suitable treatment can be developed based on the results from this analysis and can 
assess the health of the patients by measuring the parameters. There are objective and semi-subjective techniques which give information about critical parameters for rehabilitation.

Table 3 Illustrates the importance of gait parameters in various applications

\begin{tabular}{ll}
\hline Gait sub phase & Interval (of gait cycle) \\
\hline Initial contact & $0 \%-2 \%$ \\
Loading response & $2 \%-12 \%$ \\
Mid Stance & $12 \%-31 \%$ \\
Terminal Stance & $31 \%-50 \%$ \\
Pre-Swing & $50 \%-62 \%$ \\
Initial Swing & $62 \%-75 \%$ \\
Mid Swing & $75 \%-87 \%$ \\
Terminal Swing & $87 \%-100 \%$ \\
\hline
\end{tabular}

\section{Role of wearable sensors in human gait analysis}

The primary challenge of a potential wearable system is to accurately track human movements and perform gait analysis using a single or multiple combinations of sensors. To measure different

Table 4 Overview of various wearable sensors used for Human Gait analysis characteristics of the human gait, wearable sensors are attached at various segments of a human body. This section presents the overview of different sensors that can be used in wearable tracking system. An overview of various wearable sensors used for Human Gait analysis is presented in the Table 4.

\section{Inertial sensor based human gait analysis}

Recent advancements in MEMS technology have made inertial sensors readily available with low cost, less size and highly transportable. IMUs with combination of accelerometers, gyroscopes and magnetometers are used for gait analysis in various research fields. The miniaturization of inertial sensors overcomes the limitations related to restricted range of motion and allows the possibility of integrating them on to the body to track body segment orientation, position for human gait analysis. Companies such as XSens Technologies and Inn labs are providing inertial sensing solutions from daily activities to research related ones. Table 5 provides an overview of various types of instrumentation used for different experiments. ${ }^{44}$ Details included in the table regarding the type of sensors used ranged from uniaxial to triaxial accelerometers, gyroscopes and magnetic sensors. Scale of accelerometers ranged from $3 \mathrm{~g}$ to $10 \mathrm{~g}$. Gyroscopes ranged from $300-$ 1200 degree/second. Size of sensors ranged from $20 * 10 * 7.2 \mathrm{~mm} 3$ to $64 * 62 * 26 \mathrm{~mm} 3$.

\begin{tabular}{|c|c|c|c|}
\hline Application & Sports & Clinical & Recognition \\
\hline $\begin{array}{l}\text { Gait } \\
\text { Parameters }\end{array}$ & $\begin{array}{l}\text { Stride velocity, Step length, Stride length, } \\
\text { Step width, Cadence, Step Width, Step Angle, } \\
\text { Traversed distance, Accumulated altitude, Gait } \\
\text { phases, Ground reaction forces, Route, Body } \\
\text { segment orientation, Route, Joint angles, Muscle } \\
\text { force, Momentum, Body posture, Body segment } \\
\text { orientation }\end{array}$ & $\begin{array}{l}\text { Step time, Swing time, Stance time, Stop duration, Existence } \\
\text { of tremors, Fall, Stride velocity, Step length, Stride length, } \\
\text { Step width, Cadence, Step Width, Step Angle, Gait Autonomy, } \\
\text { Traversed distance, Accumulated altitude, Gait phases, } \\
\text { Ground reaction forces, Route, Body segment orientation, } \\
\text { Route, Joint angles, Muscle force, Momentum, Body posture, } \\
\text { Body segment orientation }\end{array}$ & $\begin{array}{l}\text { Stride velocity, } \\
\text { Step length, Stride } \\
\text { length, Step width, } \\
\text { Cadence, Step } \\
\text { Width, Step Angle, } \\
\text { Body segment } \\
\text { orientation, Gait } \\
\text { phases }\end{array}$ \\
\hline
\end{tabular}

Table 5 An overview of various types of instrumentation used for different experiments

\begin{tabular}{|c|c|}
\hline $\begin{array}{l}\text { Wearable } \\
\text { sensors }\end{array}$ & Description \\
\hline $\begin{array}{l}\text { Pressure and Force } \\
\text { Sensors }\end{array}$ & $\begin{array}{l}\text { These sensors are useful in measuring ground reaction forces (GRF) under the foot. These sensors are embedded in } \\
\text { foot sole to measure GRF during the gait. The most commonly used models are resistive piezoelectric, capacitive, }{ }^{31} \\
\text { strain gauged }{ }^{32,33} \text { and piezo resistive sensors. }\end{array}$ \\
\hline Goniometers & $\begin{array}{l}\text { The flexible Goniometers measure the relative rotation between two body segments. Goniometers can be classified } \\
\text { in to strain gauge, }{ }^{34,35} \text { mechanical flexible inductive and optical fiber Goniometers. }{ }^{36,37} \text { At present, numerous flexible } \\
\text { electro Goniometers are available in market. }\end{array}$ \\
\hline $\begin{array}{l}\text { Electromagnetic } \\
\text { Tracking System } \\
\text { (ETS) }\end{array}$ & $\begin{array}{l}\text { This system is based on the principle of Faraday's law of magnetic induction. In ETS, controlled magnetic fields are } \\
\text { generated from the transmitters and are detected by the receivers which are fixed on the moving object. Various } \\
\text { commercialized ETSs are used in bio-engineering field for kinematic study of body segments. }{ }^{38}\end{array}$ \\
\hline Ultrasonic Sensors & $\begin{array}{l}\text { Ultrasonic sensors are used in evaluation of gait parameters like stride length, short step. }{ }^{39,40} \text { It is also possible to use } \\
\text { these sensors in measuring the distance between floor and the foot. }\end{array}$ \\
\hline $\begin{array}{l}\text { Electromyography } \\
(\text { EMG) }\end{array}$ & $\begin{array}{l}\text { EMG is used to measure small change in current produced by muscles during contraction. EMG signals can be used to } \\
\text { measure different gait parameters. For instance, EMG signals can give information related to relative muscle tension as } \\
\text { EMG amplitude increases with the walking speed. }{ }^{4 l}\end{array}$ \\
\hline Sensing Fabric & $\begin{array}{l}\text { Most commonly used sensing fabric materials are piezoelectric, }{ }^{42} \text { piezo resistive }{ }^{43} \text { and piezo capacitive. }{ }^{44} \text { This is more } \\
\text { flexible and comfortable compared to other wearable sensors in measuring human movements. }\end{array}$ \\
\hline Inertial Sensors & Inertial sensors are the electronic devices that sense orientation and position of moving object. \\
\hline
\end{tabular}




\section{A brief review of inertial sensor algorithms}

In most of the studies using inertial sensors, the accelerometer and gyroscope output is combined in wearable sensor system. Orientation and position of the body segment is obtained by integrating angular rate from gyroscopes and translational acceleration from accelerometers. There are many issues related to integration of inertial measurements. A technique was adapted to measure knee joint angle directly by measuring data directly from eight uniaxial accelerometers attached to subject's thigh and leg without integration. Here, in this study knee was modeled as hinge joint allowing planar rotation. The limitation of this study was that the system was accurate for low speed movements. ${ }^{45}$ Knee flexion/Extension angle was calculated using measurements from 2 IMUs with accelerometer and gyroscopes attached to thigh and shank. Taking difference between thigh and shank tilt angles, knee joint angle was calculated. ${ }^{46}$. Some researchers improved this process by integrating measurements from gyroscopes and accelerometer. ${ }^{47,48}$ Gyroscopes output drifted in measurements as the time period increased. In order to correct the drift from gyroscope, a Kalman filter was built. ${ }^{49}$ The filter worked on the errors between the orientations estimates from gyroscope and gravimetric tilt sensor. This technique faced limitations when there was a large acceleration. This acceleration degraded the performance of the tilt sensor and showed large errors in orientation estimates. Taking this problem in to consideration, in another paper, drift correction was performed only at stationary periods assuming accelerometer sensed only gravitational acceleration. ${ }^{50}$

Some researchers tried to get orientation estimate by integrating angular rate from gyroscope and inclination information from accelerometers. In their system, the integration was done around the vertical axis. The difference between gyroscope and accelerometer measurements was used as an input to the Kalman filter to obtain more accurate orientations in next step. ${ }^{51}$ However, the estimates were accurate only for short periods when the subject was stationary and acceleration was only due to gravity. Other researchers used multiple accelerometers without gyroscopes to obtain limb orientation and position. ${ }^{52}$ For a short observation interval, there was an error of $10 \mathrm{deg}$ in orientation estimates. Therefore, they combined accelerometer and gyroscopes to track position and orientation during various tasks. Estimation errors became minimal. But, the limitation was that this was applicable only for simple movement based tasks. Some implemented quaternion based complimentary filter to reduce the drift from gyroscopes. ${ }^{53}$ In this system, a triaxial accelerometer, gyroscope and magnetometer were embedded in a sensor module and used for experimentation. In a subsequent study, MARG (Magnetic, Angular Rate and Gravity Sensor) module was implemented for orientation tracking. Errors were mainly due to magnetic field disturbances and ranged from $12 \mathrm{deg}$ to $16 \mathrm{deg} .{ }^{54}$ They proposed that these errors can be minimized by maintaining less distance from the source. Therefore, this limited the range of motion and was restricted to certain environments only. In order to reduce the magnetic disturbances, a Kalman filter was developed which had two inputs, one related to inclination difference between the accelerometer and gyroscope and other related to inclination difference between gyroscope and magnetometer. ${ }^{55}$ This model based sensor fusion algorithm showed significant improved results in orientation estimates. In a further study, a complimentary Kalman filter with accelerometers and gyroscopes was combined with body mounted magnetic system. This tracker was tested in the region closer to magnetic materials and displayed good results. In order to evaluate gait parameters like stride length, walking speed IMU was attached to the foot and a system was developed using biaxial accelerometer and gyroscope to perform gait analysis. ${ }^{56}$ Based on the pitch angle obtained from gyroscope, gait transition phases were evaluated. The limitation was that they assumed the motion is planar and in the sagittal plane only. In a subsequent study, Extended Kalman filter tracker was constructed using the quaternion based configuration with triaxial accelerometers, gyroscopes, magnetometer for orientation tracking. ${ }^{57}$ In a study, a system was developed using MARG sensors and quaternion based EKF to track human body segments orientation. The authors reported an error of $9 \mathrm{deg}$ for roll angle when the subject was moving. ${ }^{58}$ In a further study, they presented a simplified algorithm for 3 DOF orientation estimates using only accelerometers and magnetometers. ${ }^{59}$

Researchers used wearable inertial sensors containing gyroscopes and accelerometers to tack human arm movement in a rehabilitation system. Series of tasks were performed on the subjects and results showed high correlation between estimates obtained from inertial and optical tracking system. ${ }^{60,61}$ In a study, a portable inertial tracking system was constructed using six IMUs. They tested their system for tasks during stair climbing. ${ }^{62}$ They validated the algorithm with optical tracking system and showed root mean square error of $4 \mathrm{deg}$. The limitation of this study was that this method was repeated for calculation flexion/extension angles around single axis. Some used wearable inertial sensors to estimate thigh, shank and knee angles. In order to reduce the sensor drift in gyroscopes, some researchers imposed biomechanical model which included physical constraints in to range of motion.

\section{Coupling of multiple IMUs for human gait analysis}

Multiple IMUs strapped on various parts of the body allow gathering complex data about the body segments and can estimate the kinematics using the acquired data. In order to calculate joint angles at various segments of the body, multiple IMUs are used in conjunction and measured data is fused. For an instance, to calculate knee joint flexion/extension angle, IMUs at the thigh and shank are used. There are two popular methods for joint angle measurement; the first method assumes that each IMU provides highly accurate estimates of orientation with respect to common fixed coordinate system. By taking local joint coordinate axis and orientation together, flexion/ extension can be calculated. This approach can be seen in L'Hermette M. ${ }^{13,16,63}$ The second method was to integrate both angular rates around the joint axis which reduces the drift to yield more accurate angle. In a study, spatial-temporal parameters of gait were estimated using three IMUs attached to subject's thigh and shank. ${ }^{47}$ Takeda et al. ${ }^{16}$ proposed a setup with 7IMUs. These IMUs were strapped on hip, left and right thigh, shank and foot. They developed a wavelet based method to estimate the inclination of the sensors. The second method used joint position vectors and did not rely on magnetometer. There are several sensor fusion algorithms to couple multiple IMUs with other sensors are available in literature. The most popular ones are Virtual IMU observation fusion, Centralized filter fusion, Federated Filter Fusion implementation (Figure 2). 


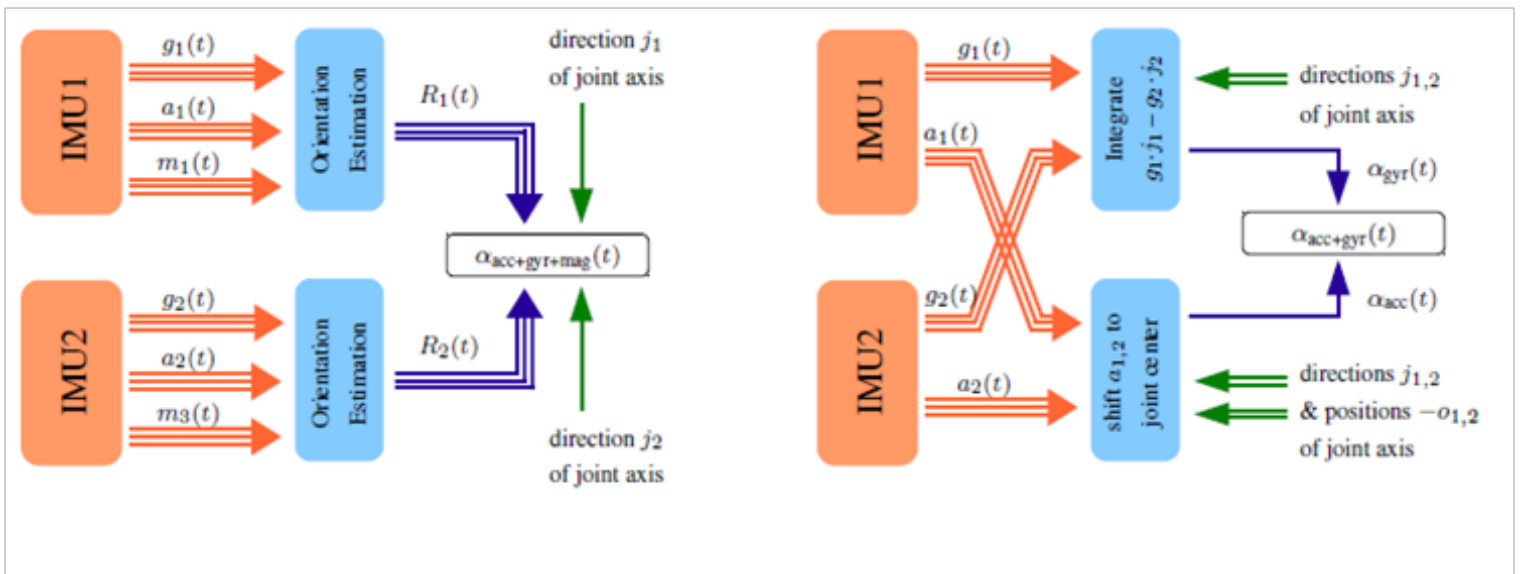

Figure 2 Two different methods for joint angle measurements using multiple IMUS. ${ }^{19}$

\section{Applications}

Small, low cost and low power wearable sensors can be used as a tool for gait analysis. Gait analysis using wearable sensors has various applications in the fields of clinical, biomechanical, biomedical, sports, rehabilitation and so on.

\section{Bio-medical applications}

In recent years, researchers and clinicians have used inertial tracking in medical field related to rehabilitation and to gage progress of treatment etc. Motion capture and analysis has also been used to detect body posture and postural instability in elderly subjects. To assess disorders and motor impairments, clinicians regularly uses tracking technologies.

\section{Sports science}

Inertial tracking is the preferred analysis method in a wide range of sports applications, physical education and practice. Physical limitations and movement optimization are of great interest to researches, athletes and coaches. Motion tracking allows them to learn about injury mechanisms and preventions. It can also be used to improve player's technique for better results in sports applications. An athlete's performance can be improved using an ambulatory gait analysis technique, which can promote performance improvement. A number of studies have used gait analysis in the sport events related to running, ${ }^{40}$ golf, ${ }^{64}$ and baseball training..$^{65}$ These are also used for an exercise coaching. ${ }^{66}$ Therefore, combining gait analysis with sport training can correct improper posture, motion and can assess the performance of the athlete.

\section{Clinical and health monitoring}

An important application of gait analysis using wearable sensors is fall risk estimation. This type of accident is most commonly seen in elderly people. A study analyzed and reported the main reasons for falling and the gait phase, where this occurs more frequently. ${ }^{67}$ Some studies developed algorithms to judge emergency fall. ${ }^{68-70}$ Bourke Lyons \& Bourke et al. ${ }^{68,69}$ determined the acceleration threshold for fall detection using triaxial accelerometer attached at the waist and head. The results for this study proved that the gait analysis can be applied to the fall detection and fall risk estimation in daily life.
Ambulatory gait analysis becomes necessary for the patients with Parkinson's or osteoarthritis. ${ }^{71}$ For the detection of gait abnormalities and assessment of the recovery, gait analysis can be applied.

\section{Limitations of previous studies}

a. Most of the studies employed strap down integration of angular rates. The drift in the inclination of the IMU's orientation is eliminated by assumption that the measured acceleration is only due to gravity.

b. In most of the studies, the researchers integrated angular velocity information from gyroscope with the inclination information from accelerometer in to a Kalman filter to reduce drift. Orientation estimates were accurate only for short periods when the subject was stationary as acceleration aiding was only due to gravity.

c. In other studies, magnetometers were used to update the inclination information around the vertical axis. But, these led to errors due to magnetic disturbances.

d. Kalman filter for the prediction of orientation estimation in highly nonlinear dynamics could lead to large errors.

e. In some papers, an extension to Kalman filter EKF (Extended Kalman Filter) was implemented to linearize the process and measurement models. This linearization led to errors in nonlinear dynamics. This method also required high computational time and efficiency and very much prone to errors.

f. The strategy of implementing tracking algorithms with the use of large number of sensors led to large computational cost and prone to stability problems as number of sensors increased the state and observer dimensions.

g. In many papers, researchers assumed that the local coordinate axis of the sensors aligns with the joint coordinate axis. This segment to sensor mounting assumptions led to errors in the long periods.

\section{Recent trends in inertial wearable sensors system for human gait analysis}

Generally, gait analysis comprises the study of kinematics in the sagittal, coronal and transverse planes. Although kinematics in 
the sagittal plane provides the most reliable information, it is only partial in clinical gait analysis. 3D intersegment moment analysis is of great interest. The transverse and coronal plane measurements are less used and commonly show less reliability. Nevertheless, the knee and ankle joint center trajectories are of interest in clinical gait analysis. ${ }^{16}$ Therefore, there is necessity to build a system which can give 3D moment analysis. Gait measurements in three axes are made by $\mathrm{H}$-Gait system which consists of magnetic and inertial sensors. ${ }^{16}$ The H-Gait system consists seven magnetic and inertial measurement units (MIMUs) and each MIMU sensor is composed of tri-axial accelerometer, gyroscope and magnetometer. In recent years, advanced algorithms are implemented on inertial sensor based gait analysis system to analyze spatiotemporal parameters for normal human (Truong \& Jeong 2016), stroke subjects (Trojaniello et al. 2015), and PD patients (Rezvanian \& Lockhart 2016, Trojaniello et al. 2015). (Rezvanian \& Lockhart 2016) employed the continuous wavelet transform (CWT) of the accelerometer data to define an index for correctly detecting the freezing of gait (FOG) of the PD patients.

The stride velocity and stride length revealed significant differences in a walking test (Trojaniello et al. 2015). Temporal gait parameters are estimated using Bayesian models on acceleration signals (LópezNava et al. 2016). A mobile gait analysis system is designed on the shoes with IMUs mounted and presents a method to estimate spatial and temporal parameters in geriatric patients (Rampp et al. 2015). In this paper, the temporal gait events were detected by searching for characteristic features in the signals. To calculate stride length, the gravity compensated accelerometer was double integrated, and sensor drift was modeled using piece-wise linear function. A novel approach is presented to estimate stride length using deep Convolutional neural network to map stride specific inertial sensor data mounted on the shoe to resulting stride length (Hannink et al. 2016). Also, Deep Convolutional neural network framework is used to estimate spatial parameters and temporal parameters in geriatric patients (Hannink et al. 2017). A phase variable approach is designed to perform gait classification which utilizes measured motion of the thigh segment provided by a single IMU (Bartlett \& Goldfarb 2017). An activity recognition system is built by using four Imus mounted on bilateral thigh and shank and smart shoes to classify three walking gait states (Chinimilli, Redkar \& Zhang 2017).

\section{Review of control strategies in exoskeletons}

The objective of this section is to address control strategies of lower limb exoskeletons. In general, control strategies need to deal with issues related to user-exoskeleton mutual interaction in power and information exchange. A potential exoskeleton with effective control strategy should be able to deliver effective mechanical power to exoskeleton based on kinematics and kinetics information. Physical interaction depends on low level controller and cognitive transfer depends on high level controller.

\section{Sensitivity amplification control}

Sensitivity Amplification Control is mostly used in projects where objective is to augment load carrying capacity of the user. This controller calculates the desired joint positions and torques directly. The controller depends on inverse dynamics model and the force exerted by the user on the exoskeleton. This information is given as a positive feedback to the controller and is controlled by scaling amplification parameters. However, the controller could amplify an external disturbance due to which the system may become unstable.
Therefore, user needs to reach a new stable condition of the whole system. This strategy requires highly accurate inverse dynamics model. This strategy is implemented in BLEEX, ${ }^{73}$ NAEIES. ${ }^{74}$

Berkeley lower extremity exoskeleton (BLEEX): The control strategy implemented in BLEEX is based on sensitivity amplification controllers. $^{72}$ The control algorithm in BLEEX ensures that exoskeleton always moves in sync with the user. This scheme is particularly effective when the contact location between the user and exoskeleton is unpredictable. In order to move the user, the controller must give the exoskeleton a large sensitivity to the small torques applied by the user. The exoskeleton controller uses the inverse dynamics of the exoskeleton to achieve this. The sensitivity amplification control strategy in BLEEX models the torque applied by the human user on the exoskeleton as d. Neglecting gravity, the angular velocity is modeled as

$$
V=G r+S d
$$

$\mathrm{S}$ is the transfer function or sensitivity from human torque to exoskeleton angular velocity, $r$ is the actuator input and $G$ is the transfer function from actuator inputs (Figure 3). The objective is to maximize sensitivity to $d$ without direct measurement. Sensitivity amplification achieves this by creating a feedback loop from a controller (C). Sensitivity equation changes to Snew after applying positive feedback

$$
\text { Snew }=\frac{v}{d}=\frac{S}{(1+G C)}
$$

In order to provide larger sensitivity, BLEEX uses $\alpha$ (scalar amplification factor) from

$$
C=\left(1-\alpha^{\wedge}(-1)\right) G^{\wedge}(-1)(3)
$$

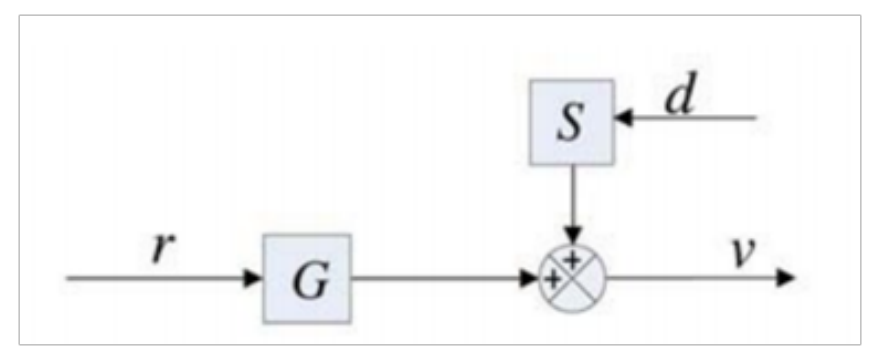

Figure 3 The exoskeleton's angular velocity is shown as a function of the input to the actuators and the torques imposed by the pilot onto the exoskeleton. ${ }^{72}$

The upper loop shows how its pilot moves the exoskeleton through applied forces. The lower loop shows how the controller drives the exoskeleton. ${ }^{72}$ In the case of BLEEX, designers ignored disturbance rejection in order to maximize the response of the suit of the user. Users should take action to balance out the disturbances and stabilize the system. BLEEX uses information from 16 accelerometer and 8 encoders to determine angle, angular acceleration and angular velocity of 8 actuated joints. Each single axis force sensor provides measurements to perform low level control at each actuator. Applying this sensitivity amplification control scheme (Figure 4), BLEEX has achieved successful walking at $1.3 \mathrm{~m} / \mathrm{s}$ with a $34 \mathrm{~kg}$ payload. ${ }^{72}$ 


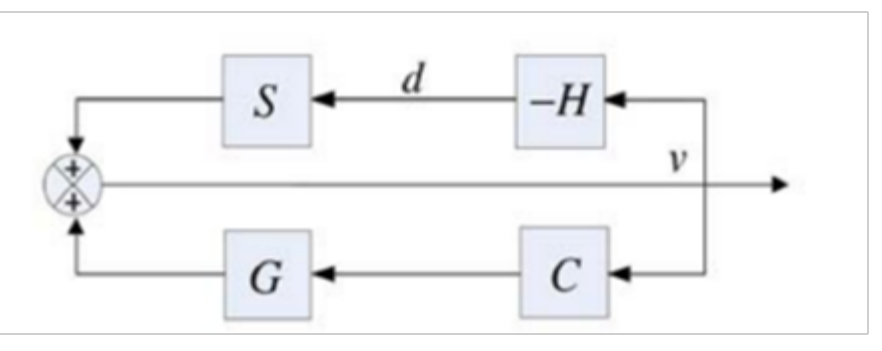

Figure 4 This block diagram shows how an exoskeleton moves.

Assessment of sensitivity amplification control: Sensitivity Amplification strategy is currently one of the best in class strategy. The process and hardware are simple yet effective. However, the approach has several disadvantages including the need for high reliability and accurate model of exoskeleton and the fact that sensitivity amplification will amplify disturbances. In highly dynamic cases, it becomes very critical that sensitive amplification will amplify forces acting on the user suit and operator will have to adjust actions to compensate. The possible solution can be filter out the external disturbances, but it is not possible with additional sensory equipment. It is important to note that this type of approach would present its own challenges and limitations in sensing and calibration of current EMG systems.

\section{Model based control}

This strategy mainly depends on accuracy of the model which requires measurements from the group of sensors to capture kinematics and dynamic variables. The desired action is computed from the model considering gravity compensation, Zero Moment Point (ZMP) balance criterion. Therefore, under a model-based control, the desired robotic action depends on user-exoskeleton model. This control strategy is adopted in projects $\mathrm{HAL},{ }^{74} \mathrm{ABLE},{ }^{75} \mathrm{BE},{ }^{76} \mathrm{WWH},{ }^{77}$ $\mathrm{WPAL}^{78}$ and XOR. ${ }^{79}$

ABLE: It consists of a powered lower-limb outhouses, a pair of hand crutches and a pair of foot mobile platforms to assist the user in standing pose and stair ascending. ${ }^{76}$ The desired forces and torques are determined from quasi-static human-ABLE model. ABLE is a force transfer system for impaired lower limbs subjects. Cooperation between user and the orthosis is defined according to different maneuvers.

Hybrid assistive limb: HAL is developed to help the users executing daily activities. Model based control strategy is implemented in HAL to support knee flexions for the persons with motion difficulties. ${ }^{74}$ The total knee flexion is a combination of three components: an assistive torque to drive the knee joint, a viscous torque providing damping effect and a gravity compensating torque calculate from static-lower limb HAL human model. The control strategy is a model based approach which estimates human intention from sEMG activity and provides power to augment torque provided by the operator. User intention is identified by thresholding sEMG sensor data and a relative autonomous torque assist strategy is built. This approach provides a knee torque response which includes a combination of assistive torque component, viscous damping component that reduces high velocity motion for safety and a gravity compensation torque.

\section{Adaptive oscillators based control}

An adaptive based oscillator was first developed by, in order to synchronize with the instantaneous phase and frequency of any periodic signal. This concept is implemented in wearable robots

with the objective of capturing bio-signal features in walking. The limitation of this strategy is that it is applicable to subjects which deliver periodic and stable signals. This strategy is implemented in Full body Exoskeleton by Matsubara et al., ${ }^{80}$ Robot uit by Zhang $X$ \& Hashimoto ${ }^{81}$ and Lower limb exoskeleton by Sergi, Accoto et al. ${ }^{82}$ Typically, the building blocks for the adaptive oscillators based control system consists of the dynamic model, coupled Adaptive Frequency Oscillator (AFO) which includes signal estimator and the torque estimator and the human torque (Figure 5). Figure 6 shows the block diagram of the combined system implemented in Ajayi MO et $a^{184}$ The idea related to couple frequency oscillator was used to define a precise way of learning periodic input signal. This approach was used to learn non-sinusoidal periodic signals due to the fact that most of the human movements are non-sinusoidal in nature. This coupling scheme, which is more than a just a dynamic Fourier series decomposition of non-sinusoidal periodic signal was first proposed in Righetti L et al. ${ }^{85}$

human

cyclical movement
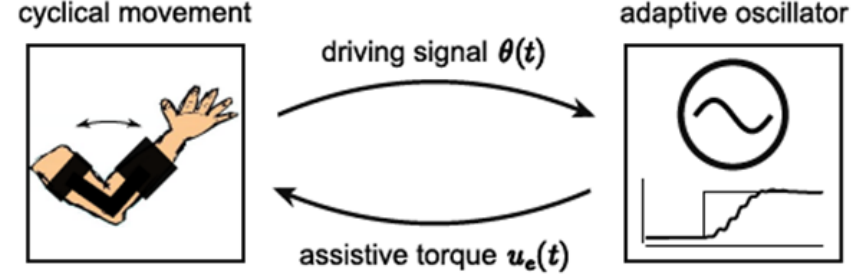

Figure $\mathbf{5}$ Sketch of the synchronization between the human joint (elbow in this case) and adaptive oscillator. ${ }^{83}$

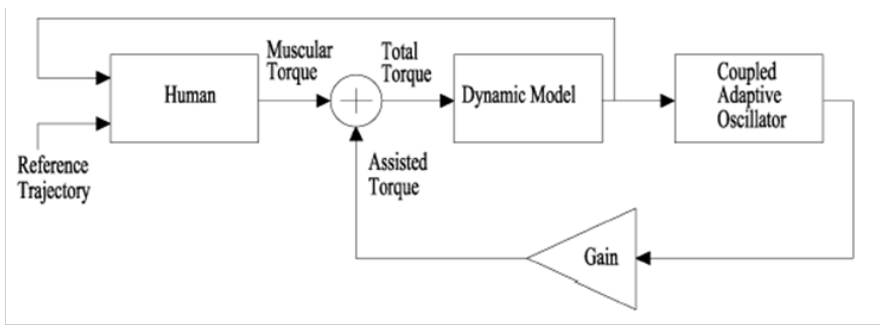

Figure 6 Block diagram of the human knee and ankle + orthosis. ${ }^{84}$

\section{Predefined gait trajectory control}

In predefined gait trajectory strategy, the desired joint angle is prerecorded from a healthy person and then replayed on an exoskeleton. For the robustness of the controller, desired joint trajectory is parameterized from different postures. This kind of strategy is usually targeted towards gait disordered patient losing normal voluntary movements. This strategy is adopted in projects ATLAS,${ }^{86} \mathrm{HAL}^{87}{ }^{87} \mathrm{IHMC}^{6}{ }^{6}$ MINDWALKER,${ }^{88}$ ReWalk, ${ }^{89}$ eLEGS. ${ }^{90}$ This approach (Figure 7) has three main drawbacks:

a. user will be moved anyway even if he stays passive, user's own effort is not promoted.

b. The reference trajectory is obtained or captured from the gait pattern of one or several individuals and might therefore not correspond to a suitable trajectory for the patient being trained.

c. This strategy is prone to errors. The position-based control approach is not suitable for walking assistance application due to several realistic problems. 


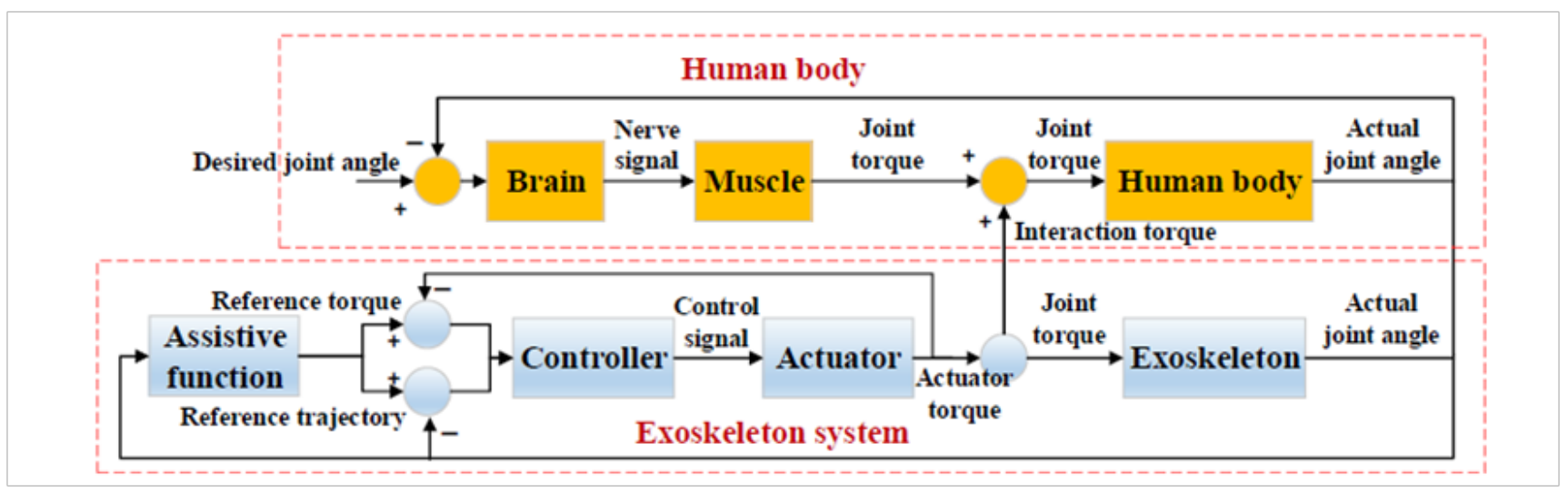

Figure 7 Schematic of the human-exoskeleton cooperation system. ${ }^{91}$

Human gaits vary greatly depending on the factors such as weight, age and anthropometry. Therefore, it is very difficult to predefine the motion trajectories which will match the different individuals walking gaits. In training trajectory control, the predefined trajectories of the lower limb joints usually collected from healthy individuals are used as the control targets. However, by using this kind of control, the wearers are passively trained to follow a predefined reference trajectory and their initiatives or motivations are not usually considered.

Hybrid assistive limb: The main aim of HAL is to provide healthy users with augmented physical strength and assist gait disordered people with movement. The hip and knee joints of HAL are controlled on the basis of two gait phases: Swing and Support phase. The desired trajectories of the joint are prerecorded from a healthy subject and are replayed these two phases by using a real time intention estimator. This control strategy is validated with two subjects: lower limb sensory paralysis user and healthy user. Feasibility and reliability of the whole system are presented in Suzuki K et al. ${ }^{92}$

ATLAS: ATLAS aims to deploy an active orthosis for gait assistance for children suffering from quadriplegia. ${ }^{86}$ Initially, considering the target end user, ATLAS aimed to be a total autonomous assistive orthosis which gives maneuver triggers to the wearer. In the later stages, ATLAS employed two finite state machines to separately tune the hip and knee joint trajectories from the healthy subject quadriplegia. $^{86}$

Mind walker: Mind walker is developed for paraplegics to regain locomotion capability. Six healthy and four SCI participants were involved in ground level walking experiments. ${ }^{88} \mathrm{EMG}$ patterns of their upper limb muscles were measured and showed to be augmented for stepping. In healthy subjects, EMG activities of leg muscles were similar or even larger during exoskeleton walking operation.

Predefined gait trajectory on gait pattern control: This strategy is different from predefined gait trajectory control as system here continuously tracks predefined joint trajectory and controls the device to act synchronically with expected gait events. Here the exoskeletons provide assistance based on passive strings or pneumatic cylinders with impedance or compliance controlled by means of the activation of the elements.

\section{Fuzzy controller}

This is one of the interesting strategies used in controlling exoskeletons. This strategy is typically used when it is difficult to find an accurate dynamic model. A fuzzy controller consists of four main blocks: Fuzzification block, Fuzzy rules block, Interface and Defuzzification block. Inputs are fuzzified and certain rules are implemented based on the knowledge of the system in the rules block and Defuzzification block coverts back fuzzy results in to crisp output signals. This controller requires manually tuning of parameters for specific tasks. It is implemented in projects like, ${ }^{93}$ EXPOS. ${ }^{93,94}$ Recently researchers ${ }^{95}$ proposed a preliminary algorithm for active assistance. The block diagram of the active controller is represented in Figure 8. The desired hip joint velocity can be estimated as follows:

$$
\omega \text { Hip }=K_{\text {front }} \frac{d F_{\text {front }}}{d t}+K_{\text {rear }} \frac{d F_{\text {rear }}}{d t}
$$

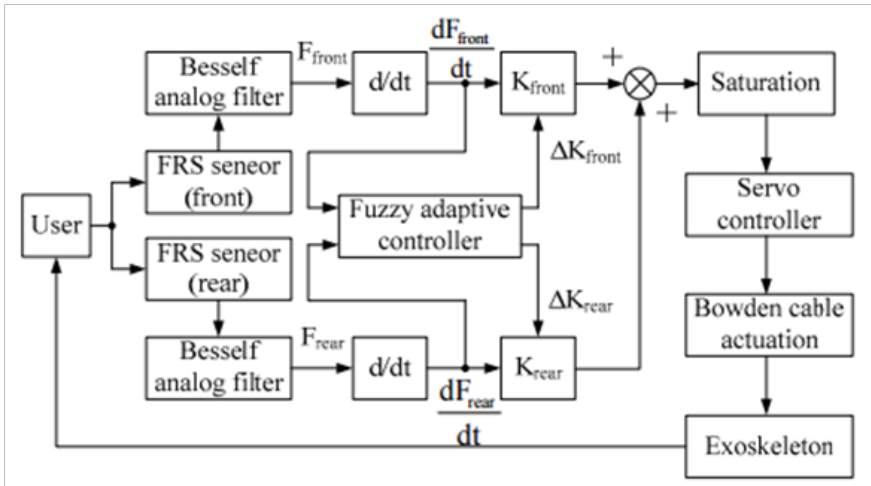

Figure 8 The block diagram of the active control algorithm implemented on each joint of the exoskeleton. ${ }^{95}$

Where $\mathrm{F}_{\text {front }}$ and $\mathrm{F}_{\text {rear }}$ denote the pressure values on the front side and rear side of the fastening strap. $\mathrm{K}_{\text {front }}$ and $\mathrm{K}_{\text {rear }}$ are the parameters to adjust the scale of joint velocity. Therefore, to improve the assistance performance, a fuzzy adaptive controller is developed based on the Mamdani model and applied to the control system. Fuzzy turning 
rules are defined with the change rates of measured pressure from the FSR sensors as the inputs. The fully populated rule consists of 25 input rule combinations.

\section{Hybrid control strategy}

Hybrid controller takes the merits of different strategies and combines into a model to control the exoskeleton. For example, BLEEX adopts sensitive amplification controller in swing phase and a position controller in the stance phase. A hybrid controller was implemented using this model based controller in stance phase and a predefined trajectory controller (Figure 9) in swing phase. AIT leg exoskeleton predefines the gait trajectory offline and uses a fuzzy controller to adjust the trajectory online. This strategy is implemented in projects BLEEX ${ }^{96}$ and AIT. ${ }^{97}$ HAL exoskeleton used two types of control systems designed for two different applications. For gait assistance and rehabilitation, an autonomous control system that carried user through predefined gait trajectories by controlling knee and hip joints. The exoskeleton drove the user to follow pre-recorded joint trajectories. For human strength augmentation, model-based approach was used which estimated human intention through sEMG's (surface EMG in Figure 10) devices and provided power to augment torque.

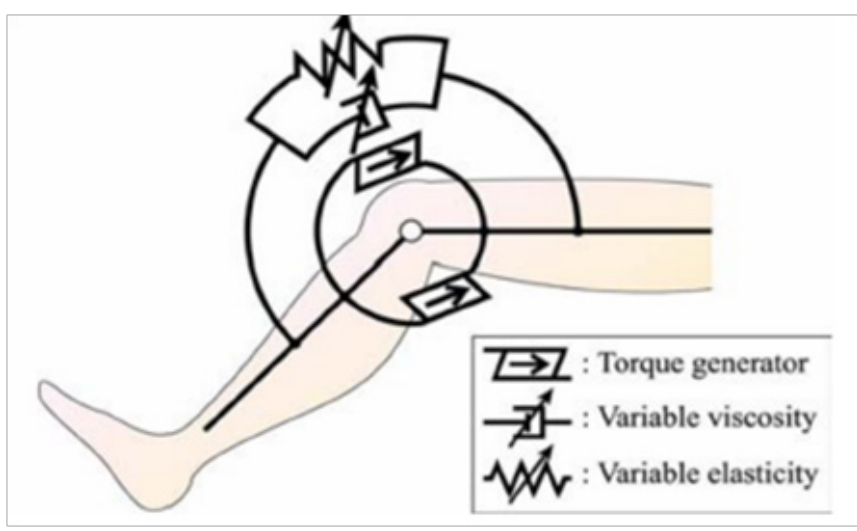

Figure 9 Model of operator's muscle group knee joint. Arrows in this figure mean contraction directions (Predefined gait trajectory control is used). ${ }^{5}$

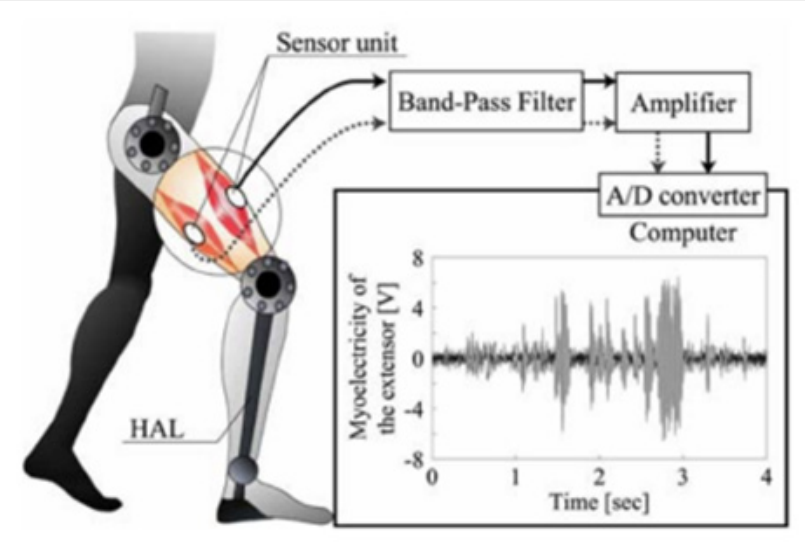

Figure 10 Process of measuring my electricity (A model based control approach is used). ${ }^{5}$

\section{Control strategies for specific tasks}

Several strategies are proposed and adopted for different targeted tasks. For the people with gait disorders, predefined trajectory control is used. The joint trajectory is prerecorded from a healthy person or extracted from clinical experiments. Parameterization of joint trajectories is done with user's motion and gait phases. In the literature, most of the control strategies are focused on providing assistance in sagittal plane. Only very few are able to focus on the stability issues addressing hip movements. A model based control strategy is very simple and widely used technique for healthy, disabled or for loading, capability augmentation. As this strategy totally depends on human exoskeleton model, kinematics need to be properly modeled. It is always difficult to get an accurate model due to multi body dynamics interaction. On the other hand, adaptive oscillator based control provides continuous gait phase by synchronizing joint angles or other periodic bio signals. This kind of strategy can generate periodic gait signals. Fuzzy controller can produce desired motion by merging different bio-mechanical signals. The limitation of this strategy is that the parameters need to be tuned manually as implementation takes more time and the computationally is very costly. For the projects aimed at augmenting load carrying capacity, sensitivity amplification control strategy is very effective. There are also devices which implements hybrid control strategy taking advantage of multiple strategies at specific stages of control Table 6 . The reviewed strategies are presented in the Table 7, Figure $11 \& 12$ show the exoskeletons described in Table 6.

\section{Identification of gait patterns to develop controller algorithm}

A Gait Identification method for an exoskeleton presents the gait sub-phases in coordinated human-machine motion. DARPA has performed many experiments since 2000 in order to improve soldiers load carrying capacity. Along with mechanical structure, control and power supply gait identification plays a crucial role in exoskeleton design ${ }^{116}$ made use of s-EMG feature signal of the lower limb to identify gait patterns with cerebral palsy. Researchers ${ }^{117}$ used fusion method to combine information from ankle angle and forces of both feet to establish gait prediction model. This motion law was learned and trained by machine learning algorithms. Researchers ${ }^{118}$ also used SVM (Support Vector Machine) method to identify the upper limb movements by the s-EMG features ${ }^{119}$ used four foot piezoelectric switches and an integrated electronic circuit for human gait identification in the stance stage of walking duration. Five tape switch sensors were used in the shoes to identify the gait of the exoskeleton. ${ }^{120}$ This method identified seven gait phases in walking duration. Some gait identification methods included pattern recognition and neural network classifiers. ${ }^{121}$

Force plates and motion capture systems are used for identifying gait phases. ${ }^{122,123}$ Ground reaction forces are calculated by using force plates. Individual events of heel strike (HS) and Toe off (TO) be identified by applying thresholds values on the recorded force from the force plates. ${ }^{124}$ Pressure sensitive mats and foot switches provide the option of being used outside the laboratory environment. Foot switches like force sensitive resistors can be attached to in-soles of the shoe. However, studies show that they are not durable and reliable for long term use due to mechanical failures. Another disadvantage is that these sensors provides temporal information only, thus restricting the scope to analyze spatial parameters of the gait. Alternative for such long 
term applications is provided by inertial sensors like accelerometer and gyroscope. Recently, many gait identification algorithms have been developed using inertial sensors. ${ }^{124}$ Some used gyroscope ${ }^{125}$ and others used accelerometers. ${ }^{126,127}$ A major disadvantage of using inertial sensors is that they provide noisy information and thus require robust signal processing algorithms for gait analysis. For the identification of gait related events, accelerometers seem to be better choice than gyroscopes as gyroscope gives large drift errors during turning, jerks and sudden movements. Accelerometers suffer from noise due to mechanical vibrations and calibration errors but they do not diverge in time and in many cases they can be handled by signal processing algorithm effectively.

Table 6 Hybrid control strategy taking advantage of multiple strategies at specific stages of control

\begin{tabular}{|c|c|c|c|c|}
\hline \multirow{2}{*}{ Authors } & \multirow{2}{*}{ Number of sensor module } & \multicolumn{3}{|c|}{ Components of each sensor module } \\
\hline & & Accelerometer & Gyroscope & Magnetic sensor \\
\hline Andrews et al. ${ }^{98}$ & I & Uniaxial & & \\
\hline Mayagoitia et al. .99 & 8 & Uniaxial & & \\
\hline Coley et al. ${ }^{100}$ & 1 & & Uniaxial & \\
\hline Dejnabadi et al.' & 2 & Biaxial & Uniaxial & \\
\hline Heliot et al. ${ }^{.01}$ & 2 & Triaxial & & Triaxial \\
\hline Simcox et al. ${ }^{102}$ & 3 & 2 Biaxial & I Uniaxial & \\
\hline J Favre et al. ${ }^{2}$ & 2 & Triaxial & Triaxial & Triaxial \\
\hline Dejnabadi et al.' & 4 & Biaxial & Uniaxial & \\
\hline Ahmadi et al. ${ }^{103}$ & 3 & Triaxial & & \\
\hline O’Donovan et al. ${ }^{17}$ & 2 & Triaxial & Triaxial & Triaxial \\
\hline Mamizuka et al." & I & Triaxial & & \\
\hline J Favre et al. ${ }^{15}$ & 2 & Triaxial & Triaxial & Triaxial \\
\hline Kawano et al..$^{104}$ & 2 & Triaxial & Triaxial & Triaxial \\
\hline Zijlstra et al. ${ }^{105}$ & 2 & Triaxial & Triaxial & \\
\hline Ermes et al. ${ }^{106}$ & 3 & 2 Triaxial & & I Triaxial \\
\hline Findlow et al. ${ }^{107}$ & 2 & Triaxial & Triaxial & \\
\hline L'Hermette et al. ${ }^{13}$ & I & Triaxial & & \\
\hline T Liu et al. ${ }^{10}$ & 2 & Triaxial & & \\
\hline Z. Zhang et al. ${ }^{108}$ & I & Biaxial & & \\
\hline Cooper et al. ${ }^{4}$ & 1 & Triaxial & Triaxial & \\
\hline van den Noort et al. ${ }^{12}$ & 2 & Triaxial & Triaxial & Triaxial \\
\hline Ja Favre et al..$^{14}$ & 2 & Triaxial & Triaxial & Triaxial \\
\hline Hanlon et al. ${ }^{109}$ & 2 & Biaxial & & \\
\hline K Liu et al. ${ }^{9}$ & 3 & Triaxial & & \\
\hline Chu et al. ${ }^{110}$ & I & Triaxial & Triaxial & \\
\hline Saber-Sheikh et al."'I & 2 & Triaxial & Triaxial & Triaxial \\
\hline Clark et al. ${ }^{8}$ & I & Triaxial & & \\
\hline
\end{tabular}


Table 7 Reviewed strategies are presented

$\begin{array}{ll}\text { Projects } & \text { Control strategy } \\ \text { BLEEX 72, NAEIES 73 } & \text { Model-based Control } \\ \text { HAL74,ABLE 75, BE 76,WWH 77,WPAL 78, XOR 79 Amplification Control } \\ \text { Matsubara T et al. 80,8I,II2 }\end{array}$

Figure I I BLEEX,ATLAS, HAL, Mina, MINDWALKER, ReWalk, eLEGs, Vanderbilt Lower-Limb Orthosis. ${ }^{77}$

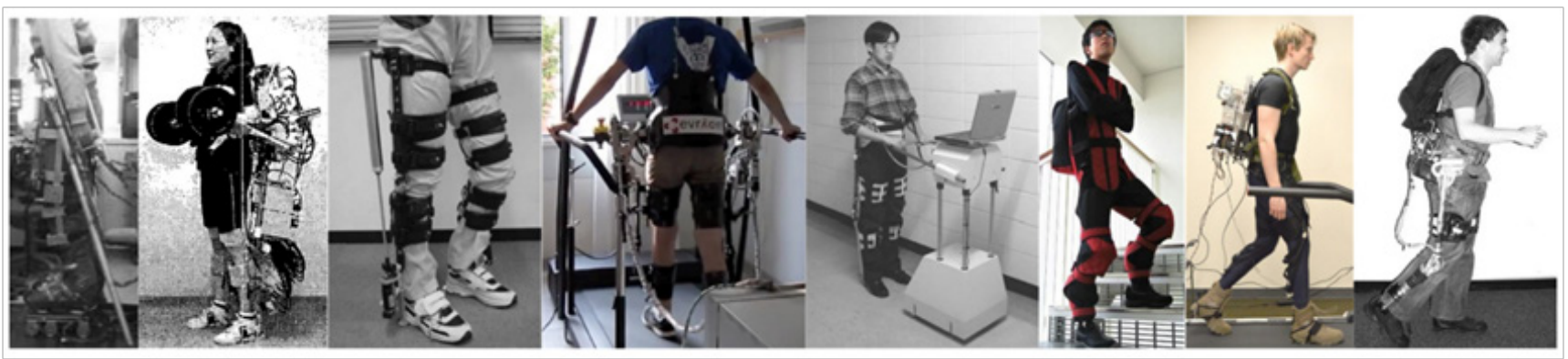

Figure I 2 ABLE, Nurse Robot Suit,WWH, Full body Extender, EPOS, Power Assist wears by D.Sasaki, Soft Exosuit, MIT Exoskeleton. ${ }^{77}$

Recently, many gait identification methods have been developed using accelerometer data alone. Some of these algorithms apply various sensor fusion techniques to analyze signals obtained from individual accelerometer axis. ${ }^{46,109,128}$ Some other methods use machine learning algorithms to train the system, but the difficulty with such algorithms is that they totally depend on the labeled data and addition of any extra parameter requires re-training of the entire algorithm. ${ }^{46,127}$ In recent years, wavelet transformation is increasingly used to develop gait identification algorithms as they are shown to be more robust among peak detection algorithms. ${ }^{127,129,130}$ Gait identification methods can be useful in exoskeleton control strategies to effectively switch between different gait phases or activities.

\section{Recent developments in control strategy for lower- limb exoskeletons}

Human-robot Interaction: In recent years, impedance based control strategy became popular for lower limb exoskeleton for gait rehabilitation and assistance applications. The effectiveness of impedance control schemes for the robotic exoskeletons have been addressed through characteristic regulation of human-robot interaction forces and motion control (Huo, Mohammed, \& Amirat, 2017; Huo Mohammed, Amirat, \& Kong, 2016; Tran et al., 2016). (Huo et al. 2017) presents a control approach of a lower-limb exoskeleton by modulating the original impedance of the swinging leg to reduce the muscular efforts. (Tran et al., 2016) developed a fuzzy based impedance control strategy to provide the assistive torques by regulating the desired impedance between the exoskeleton and a wearer's limb according to a specific motion speed. Measuring interaction dynamics and using inverse dynamics models can be effective exoskeleton control approach to assist the able-bodied human subjects. ${ }^{131-135}$ However, the nature of such methods may not be suitable for the cases of patients. If the user is unable to produce 
sufficient torques in their joints, the robot may not be successfully controlled. Therefore, taking feedback of muscle activity is essential in those cases. Therefore, control strategy is developed based on EMG feedback minimization (Durandau et al., 2017; Garate et al., 2016).

\section{Conclusion}

This paper provides a brief review on wearable inertial tracking based human gait analysis with the introduction of motion capture technologies, their limitations and comparison between wearable and non-wearable systems, types of wearable sensors, details of Instrumentation used in wearable system, review on inertial sensor algorithms and coupling of multiple IMUs for human gait analysis, Applications of wearable system in human gait analysis, limitations of previous studies are illustrated and kinematic modeling of body segments, mainly on lower limb are also described in this paper. In the later part of the manuscript, a review on various current states of art assistive strategies and their validation methods are presented. Until now, human gait analysis using wearable inertial sensors has made a great progress and shown good application prospects. In order to realize wide range of technology in our lives, some technical aspects still need to be improved, such as reliability of algorithms for kinematics and kinetics in gait analysis, development of low cost integrated wearable sensor systems and so on. Modeling body segments exploring flexible constraints by introducing some compliance in to the model may accurately capture kinematics of the human body. Measurement techniques should not rely on any predefined calibrated postures or movements and should be able to find joint coordinates in any arbitrary position or orientation. ${ }^{136-139}$

With the rise of technology in the recent years, exoskeletons will play a crucial role in our daily life, especially for people with continuous need in locomotion activities with a desired level of autonomy. An assistive exoskeleton is able to provide assistance in various motion tasks: walking, running, ascending \& descending stairs. All the assistive strategies should be validated with different methods. Activity recognition becomes very important factor to be considered during control of an exoskeleton. Separate module for the activity recognition may increase the performance of the control in exoskeletons. There are several data mining offline computation methods are available to train and test human activity recognition system. But, it becomes difficult to implement these algorithms in real time and use the results for the control of exoskeleton. Research need to be carried in this area to augment the performance of control strategy in exoskeletons in real time. Wearable system with IMUs provides insight and reference for the assistive strategy to target desired trajectory. Finally, safe interaction with exoskeleton providing natural assistance to human is an issue that needs to be explored in detail.

\section{Acknowledgements}

None.

\section{Conflicts of interest}

No conflict of interest.

\section{References}

1. Dejnabadi H, Jolles BM, Aminian K. A new approach to accurate measurement of uniaxial joint angles based on a combination of accelerometers and gyroscopes. IEEE Trans Biomed Eng. 2005;52(8):1478-1484
2. Favre J, Jolles B, Siegrist O, et al. Quaternion-based fusion of gyroscopes and accelerometers to improve 3D angle measurement. Electronics Letters. 2006;42(11):612-614.

3. Luinge HJ, Veltink PH, Baten CT. Ambulatory measurement of arm orientation. J Biomech. 2007;40(1): 78-85.

4. Cooper G, Sheret I, McMillan L, et al. Inertial sensor-based knee flexion/ extension angle estimation. J Biomech. 2009;42(16):2678-2685.

5. Hayashi T, Kawamoto H, Sankai Y. Control method of robot suit HAL working as operator's muscle using biological and dynamical information. Paper presented at the Intelligent Robots and Systems. 2005.

6. Kwa HK, Noorden JH, Missel M, et al. Development of the IHMC mobility assist exoskeleton. Paper presented at the Robotics and Automation. IEEE International Conference. 2009.

7. Naidu D, Cunniffe C, Stopforth R, et al. Upper and Lower exoskeleton limbs for Assistive and Rehabilitative Applications. Paper presented at the Presented at the $4^{\text {th }}$ Robotics and Mechatronics Conference. 2011.

8. Clark RA, Bartold S, Bryant AL. Tibial acceleration variability during consecutive gait cycles is influenced by the menstrual cycle. Clin Biomech (Bristol, Avon). 2010;25(6):557-562.

9. Liu K, Liu T, Shibata K, et al. Novel approach to ambulatory assessment of human segmental orientation on a wearable sensor system. J Biomech. 2009;42(16):2747-2752.

10. Liu T, Inoue Y, Shibata K. Measurement of muscle motion for improving accuracy of body-mounted motion sensor. Paper presented at the Advanced Intelligent Mechatronics. 2008.

11. Mamizuka N, Sakane M, Kaneoka K, et al. Kinematic quantitation of the patellar tendon reflex using a tri-axial accelerometer. $J$ Biomech 2009;40(9):2107-2111.

12. van den Noort JC, Scholtes VA, Harlaar J. Evaluation of clinical spasticity assessment in cerebral palsy using inertial sensors. Gait Posture. 2009;30(2):138-143.

13. L'Hermette M, Savatier X, Baudry L, et al. A new portable device for assessing locomotor performance. Int J Sports Med. 2008;29(4):322-326.

14. Favre J, Aissaoui R, Jolles BM, et al. Functional calibration procedure for 3D knee joint angle description using inertial sensors. $J$ Biomech. 2009;42(14):2330-2335.

15. Favre J, Jolles BM, Aissaoui R, et al. Ambulatory measurement of 3D knee joint angle. J Biomech. 2008;41(5):1029-1035.

16. Takeda R, Tadano $\mathrm{S}$, Natorigawa $\mathrm{A}$, et al. Gait posture estimation using wearable acceleration and gyro sensors. J Biomech. 2009;42(15):24862494.

17. O'Donovan KJ, Kamnik R, O'Keeffe DT, et al. An inertial and magnetic sensor based technique for joint angle measurement. $J$ Biomech. 2007;40(12):2604-2611.

18. Ferrari A, Cutti AG, Garofalo P, et al. First in vivo assessment of "Outwalk": a novel protocol for clinical gait analysis based on inertial and magnetic sensors. Med Biol Eng Comput. 2010;48(1):1-15.

19. Seel T, Raisch J, Schauer T. IMU-based joint angle measurement for gait analysis. Sensors. 2014;14(4):6891-6909.

20. O'Brien JF, Bodenheimer RE, Brostow GJ, et al. Automatic joint parameter estimation from magnetic motion capture data. Proceedings of Graphics Interface. 2000. p. 1-8.

21. Yabukami S, Kikuchi H, Yamaguchi M, et al. Motion capture system of magnetic markers using three-axial magnetic field sensor. Magnetics IEEE Transactions. 2000;36(5):3646-3648. 
22. Roux E, Bouilland S, Godillon-Maquinghen AP, et al. Evaluation of the global optimization method within the upper limb kinematics analysis. $J$ Biomech. 2002;35(9):1279-1283.

23. Welch $\mathrm{G}$, Foxlin E. Motion tracking survey. IEEE Computer graphics and Applications. 2002;22(6):24-38.

24. Pappas IP, Popovic MR, Keller T, et al. A reliable gait phase detection system. IEEE Trans Neural Syst Rehabil Eng. 2001;9(2):113-125.

25. Tao W, Liu T, Zheng R, Feng H (2012) Gait analysis using wearable sensors. Sensors (Basel) 12(2): 2255-2283.

26. White SC, Winter DA. Predicting muscle forces in gait from EMG signals and musculotendon kinematics. J Electromyogr Kinesiol. 1992;2(4):217231

27. Salarian A, Russmann H, Vingerhoets FJ, et al. Gait assessment in Parkinson's disease: toward an ambulatory system for long-term monitoring. IEEE Trans Biomed Eng. 2004;51(8):1434-1443.

28. Gehlsen G, Beekman K, Assmann N, et al. Gait characteristics in multiple sclerosis: progressive changes and effects of exercise on parameters. Arch Phys Med Rehabil. 1986;67(8):536-539.

29. Waters DL, Hale L, Grant AM, et al. Osteoporosis and gait and balance disturbances in older sarcopenic obese New Zealanders. Osteoporos Int 2010;21(2):351-357.

30. Muro-de-la-Herran A, Garcia-Zapirain B, Mendez-Zorrilla A. Gai analysis methods: An overview of wearable and non-wearable systems, highlighting clinical applications. Sensors. 2014;14(2):3362-3394.

31. Lacković I, Bilas V, Šantić A, et al. Measurement of gait parameters from free moving subjects. Measurement. 2000;27(2):121-131.

32. Faivre A, Dahan M, Parratte B, et al. Instrumented shoes for pathological gait assessment. Mechanics research communications. 2004;31(5):627632

33. Riener R, Rabuffetti M, Frigo C, et al. Instrumented staircase for ground reaction measurement. Med Biol Eng Comput. 1999;37(4):526-529.

34. Nicol A. A new flexible electrogoniometer with widespread applications Biomechanics. 1987;10-B:1029-1034.

35. Rowe P, Nicol A, Kelly I. Flexible goniometer computer system for the assessment of hip function. Clin Biomech (Bristol, Avon). 1989;4(2):68 72

36. Donno M, Palange E, Di Nicola F, et al. A flexible optical fiber goniometer for dynamic angular measurements. Paper presented at the Instrumentation and Measurement Technology Conference Proceedings. 2007.

37. Donno M, Palange E, Nicola FD, et al. A new flexible optical fiber goniometer for dynamic angular measurements: application to human joint movement monitoring. Instrumentation and Measurement, IEEE Transactions. 2008;57(8):1614-1620.

38. Finley MA, Lee RY. Effect of sitting posture on 3-dimensional scapular kinematics measured by skin-mounted electromagnetic tracking sensors. Arch Phys Med Rehabil. 2003;84(4):563-568.

39. Maki H, Ogawa H, Yonezawa Y, et al. An Ultrasonic Stride Length Measuring System Employing Two Digital Compasses. Biomed Sci Instrum. 2014;50:397-402.

40. Wahab Y, Bakar NA. Gait analysis measurement for sport application based on ultrasonic system. Paper presented at the Consumer Electronics IEEE $15^{\text {th }}$ International Symposium on. 2011.

41. Wentink EC, Schut VG, Prinsen EC, et al. Detection of the onset of gait initiation using kinematic sensors and EMG in transfemoral amputees. Gait Posture. 2014;39(1):391-396.
42. Kim KJ, Chang YM, Yoon S, et al. A novel piezoelectric PVDF film-based physiological sensing belt for a complementary respiration and heartbeat monitoring system. Integrated Ferroelectrics. 2009;107(1):53-68.

43. Sawhney A, Agrawal A, Patra P, et al. Piezoresistive sensors on textiles by inkjet printing and electro less plating. Paper presented at the MRS Proceedings. 2006. p. 1-11.

44. Taya M, Kim W, Ono K. Piezoresistivity of a short fiber/elastomer matrix composite. Mechanics of materials. 1998;28(1):53-59.

45. Willemsen AT, van Alsté JA, Boom HB. Real-time gait assessment utilizing a new way of accelerometry. J Biomech. 1990;23(8):859-863.

46. Williamson R, Andrews BJ. Detecting absolute human knee angle and angular velocity using accelerometers and rate gyroscopes. Med Biol Eng Comput. 2001;39(3):294-302.

47. Aminian K, Najafi B, Büla C, et al. Spatio-temporal parameters of gait measured by an ambulatory system using miniature gyroscopes. $J$ Biomech. 2002;35(5):689-699.

48. Tong K, Granat MH (1999) A practical gait analysis system using gyroscopes. Med Eng Phys. 1999;21(2):87-94.

49. Foxlin E. Inertial head-tracker sensor fusion by a complementary separate-bias Kalman filter. Paper presented at the Virtual Reality Annual International Symposium, Proceedings of the IEEE. 1996.

50. Foxlin EM, Harrington M, Altshuler Y. Miniature six-DOF inertial system for tracking HMDs. Paper presented at the Aerospace/Defense Sensing and Controls. 1998. p. 1-15.

51. Luinge HJ, Veltink PH, Baten CT. Estimating of orientation with gyroscopes and accelerometers. Technol Health Care. 1999;7(6):455459.

52. Giansanti D, Macellari V, Maccioni G, et al. Is it feasible to reconstruct body segment $3-\mathrm{D}$ position and orientation using accelerometric data? IEEE Trans Biomed Eng. 2003;50(4):476-483.

53. Bachmann ER, McGhee RB, Yun X, et al. Inertial and magnetic posture tracking for inserting humans into networked virtual environments. Paper presented at the Proceedings of the ACM symposium on Virtual reality software and technology. 2001. p. 9-16.

54. Bachmann ER, Yun X, Peterson CW. An investigation of the effects of magnetic variations on inertial/magnetic orientation sensors. Paper presented at the Robotics and Automation, 2004 IEEE International Conference. 2004

55. Roetenberg D, Luinge HJ, Baten CT, et al. Compensation of magnetic disturbances improves inertial and magnetic sensing of human body segment orientation. IEEE Trans Neural Syst Rehabil Eng. 2005;13(3):395-405.

56. Sabatini AM, Martelloni C, Scapellato S, Cavallo F (2005) Assessment of walking features from foot inertial sensing. IEEE Trans Biomed Eng. 2005;52(3):486-494.

57. Sabatini AM. Quaternion-based extended Kalman filter for determining orientation by inertial and magnetic sensing. Biomedical Engineering, IEEE Transactions. 2006;53(7):1346-1356.

58. Yun X, Aparicio C, Bachmann ER, et al. Implementation and experimental results of a quaternion-based Kalman filter for human body motion tracking. Paper presented at the Robotics and Automation, Proceedings of the 2005 IEEE International Conference on. 2005.

59. Yun X, Bachmann ER, McGhee RB. A simplified quaternion-based algorithm for orientation estimation from earth gravity and magnetic field measurements. Instrumentation and Measurement, IEEE Transactions. 2008;57(3):638-650

60. Zhou $\mathrm{H}, \mathrm{Hu} \mathrm{H}$. Inertial motion tracking of human arm movements 
in stroke rehabilitation. Paper presented at the Mechatronics and Automation, 2005 IEEE International Conference. 2005

61. Zhou H, Hu H, Tao Y. Inertial measurements of upper limb motion. Med Biol Eng Comput. 2006;44(6):479-487.

62. Bergmann JH, Mayagoitia RE, Smith IC. A portable system for collecting anatomical joint angles during stair ascent: a comparison with an optica tracking device. Dyn Med. 2009;8(1):3.

63. Van den Noort JC, Ferrari A, Cutti AG, et al. Gait analysis in children with cerebral palsy via inertial and magnetic sensors. Med Biol Eng Comput. 2013;51(4):377-386.

64. Ghasemzadeh H, Loseu V, Guenterberg E, et al. Sport training using body sensor networks: A statistical approach to measure wrist rotation for golf swing. Paper presented at the Proceedings of the Fourth International Conference on Body Area Networks. 2009. p. 1-8.

65. Ghasemzadeh H, Jafari R. Coordination analysis of human movements with body sensor networks: A signal processing model to evaluate baseball swings. IEEE Sensors Journal. 2011;11(3):603-610.

66. De Silva B, Natarajan A, Motani M, et al. A real-time exercise feedback utility with body sensor networks. Paper presented at the Medical Devices and Biosensors, 2008. $5^{\text {th }}$ International Summer School and Symposium on. 2008.

67. Jensen J, Lundin-Olsson L, Nyberg L, et al. Fall and injury prevention in older people living in residential care facilities: a cluster randomized trial. Ann Intern Med. 2002;136(10):733-741.

68. Bourke AK, Lyons GM. A threshold-based fall-detection algorithm using a bi-axial gyroscope sensor. Med Eng Phys. 2008;30(1):84-90.

69. Bourke AK, van de Ven P, Gamble M, et al. Assessment of waist-worn tri-axial accelerometer based fall-detection algorithms using continuous unsupervised activities. Conf Proc IEEE Eng Med Biol Soc. 2010. p. 2782-2785.

70. Kangas M, Konttila A, Winblad I, et al. Determination of simple thresholds for accelerometry-based parameters for fall detection. Paper presented at the Engineering in Medicine and Biology Society, 2007. 29 th Annual International Conference of the IEEE. 2007

71. Turcot K, Aissaoui R, Boivin K, et al. New accelerometric method to discriminate between asymptomatic subjects and patients with medial knee osteoarthritis during 3-D gait. IEEE Trans Biomed Eng. 2008;55(4):1415-1422.

72. Kazerooni H, Racine JL, Huang L, et al. On the control of the berkeley lower extremity exoskeleton (BLEEX). Proceedings of the 2005 IEEE international conference. 2005.

73. Yang Z, Zhu Y, Yang X, et al. Impedance Control of Exoskeleton Suit Based on Adaptive RBF Neural Network. Paper presented at the Intelligent Human-Machine Systems and Cybernetics, International Conference. 2009.

74. Kawamoto H, Taal S, Niniss H, et al. Voluntary motion support control of Robot Suit HAL triggered by bioelectrical signal for hemiplegia. Conf Proc IEEE Eng Med Biol Soc. 2010. p. 462-466.

75. Mori Y, Okada J, Takayama K. Development of a standing style transfer system"ABLE" for disabled lower limbs. Mechatronics, IEEE/ASME Transactions on. 2006;11(4):372-380.

76. Marcheschi S, Salsedo F, Fontana M, et al. Body extender: whole body exoskeleton for human power augmentation. Paper presented at the Robotics and Automation, IEEE International Conference. 2011.

77. Yan T, Cempini M, Oddo CM, et al. Review of assistive strategies in powered lower-limb outhouses and exoskeletons. Robotics and Autonomous Systems. 2015;64:120-136.
78. Chen F, Yu Y, Ge Y, et al. WPAL for human power assist during walking using dynamic equation. Paper presented at the Mechatronics and Automation, International Conference. 2009.

79. Hyon SH, Morimoto J, Matsubara T, et al. XoR: Hybrid drive exoskeleton robot that can balance. Paper presented at the Intelligent Robots and Systems, IEEE/RSJ International Conference. 2011.

80. Matsubara T, Uchikata A, Morimoto J. Full-body exoskeleton robot control for walking assistance by style-phase adaptive pattern generation. Paper presented at the Intelligent Robots and Systems, IEEE/RSJ International Conference. 2011.

81. Zhang X, Hashimoto M. Synchronization based control for walking assist suit-evaluation on synchronization and assist effect. Paper presented at the Key Engineering Materials. 2011;464:115-118.

82. Sergi F, Accoto D, Tagliamonte NL, et al. A systematic graph-based method for the kinematic synthesis of non-anthropomorphic wearable robots for the lower limbs. Frontiers of Mechanical Engineering. 2011;6(1):61-70.

83. Ronsse R, Vitiello N, Lenzi T, et al. Adaptive oscillators with humanin-the-loop: Proof of concept for assistance and rehabilitation. Paper presented at the Biomedical Robotics and Biomechatronics (BioRob). $3^{\text {rd }}$ IEEE RAS and EMBS International Conference. 2010.

84. Ajayi MO, Djouani K, Hamam Y. Analysis of Knee-Ankle Orthosis Modelling: An Inverse Dynamics Approach Using Adaptive Coupled Oscillator. Simulation, Modeling, and Programming for Autonomous Robots. 2014. p. 122-133.

85. Righetti L, Ijspeert AJ. Programmable central pattern generators: an application to biped locomotion control. Paper presented at the Robotics and Automation, Proceedings 2006 IEEE International Conference. 2006.

86. Sanz Merodio D, Cestari Soto M, Arevalo JC, et al. Control motion approach of a lower limb orthosis to reduce energy consumption. International Journal of Advanced Robotic Systems. 2012;9:1-8.

87. Sankai Y. HAL: Hybrid assistive limb based on cybernics Robotics Research. 2011.p. 25-34.

88. Wang L, Wang S, van Asseldonk EH, et al. Actively controlled lateral gait assistance in a lower limb exoskeleton. Paper presented at the Intelligent Robots and Systems, IEEE/RSJ International Conference. 2013.

89. Talaty M, Esquenazi A, Briceno JE. Differentiating ability in users of the ReWalk TM powered exoskeleton: An analysis of walking kinematics. IEEE Int Conf Rehabil Robot. 2013.

90. Strausser KA, Kazerooni H. The development and testing of a human machine interface for a mobile medical exoskeleton. IEEE/RSJ International Conference. 2011.

91. Chen B, Ma H, Qin LY, et al. Recent developments and challenges of lower extremity exoskeletons. Journal of Orthopaedic Translation. 2016;5:26-37.

92. Suzuki K, Mito G, Kawamoto H, et al. Intention-based walking support for paraplegia patients with Robot Suit HAL. Advanced Robotics. 2007;21(12):1441-1469.

93. Kong K, Jeon D. Design and control of an exoskeleton for the elderly and patients. Mechatronics, IEEE/ASME Transactions on. 2006;11(4):428432.

94. Kiguchi K, Rahman MH, Sasaki M, et al. Development of a 3DOF mobile exoskeleton robot for human upper-limb motion assist. Robotics and Autonomous Systems. 2008;56(8):678-691.

95. Wu Q, Wang X, Du F, Zhang X. Design and Control of a Powered Hip Exoskeleton for Walking Assistance. International Journal of Advanced Robotic Systems. 2006. 
96. Zoss AB, Kazerooni H, Chu A. Biomechanical design of the Berkeley lower extremity exoskeleton (BLEEX). Mechatronics, IEEE/ASME Transactions on. 2006;11(2):128-138.

97. Aphiratsakun N, Parnichkun M. Balancing control of AIT leg exoskeleton using ZMP based FLC. International Journal of Advanced Robotic Systems. 2009.

98. Andrews DM, Dowling JJ. Mechanical modeling of tibial axia accelerations following impulsive heel impact. Journal of Applied Biomechanics. 2000;16(3):276-288.

99. Mayagoitia RE, Nene AV, Veltink PH. Accelerometer and rate gyroscope measurement of kinematics: an inexpensive alternative to optical motion analysis systems. J Biomech. 2002;35(4):537-542.

100. Coley B, Najafi B, Paraschiv-Ionescu A, et al. Stair climbing detection during daily physical activity using a miniature gyroscope. Gait Posture. 2005;22(4):287-294

101. Heliot R, Pissard-Giboiiet R, Espiau B, et al. Continuous identification of gait phase for robotics and rehabilitation using microsensors. Proceedings $12^{\text {th }}$ International Conference. 2005.

102. Simcox S, Parker S, Davis GM, et al. Performance of orientation sensor for use with a functional electrical stimulation mobility system. $J$ Biomech. 2005;38(5):1185-1190.

103. Ahmadi A, Rowlands DD, James DA. Investigating the translational and rotational motion of the swing using accelerometers for athlete skill assessment. $5^{\text {th }}$ IEEE Conference. 2006.

104. Kawano K, Kobashi S, Tsumori Y, et al. Evaluation of pivot shift in the anterior cruciate ligament-injured knee using inertial and magnetic sensors. 2008.

105. Zijlstra A, Goosen JH, Verheyen CC, et al. A body-fixed-sensor based analysis of compensatory trunk movements during unconstrained walking. Gait Posture. 2008;27(1):164-167.

106. Ermes M, Pärkka J, Mantyjarvi J, Korhonen I. Detection of daily activities and sports with wearable sensors in controlled and uncontrolled conditions. IEEE Trans Inf Technol Biomed. 2008;12(1):20-26.

107. Findlow A, Goulermas JY, Nester C, et al. Predicting lower limb joint kinematics using wearable motion sensors. Gait Posture. 2008;28(1):120 126

108. Zhang Z, Wu JK, Huang Z. Gaussian particle filter for tracking hip angle in gait cycles. HealthCom. 2008.

109. Hanlon M, Anderson R. Real-time gait event detection using wearable sensors. Gait Posture. 2009;30(4):523-527.

110. Chu VW, Fong DT, Chan YY, et al. Differentiation of ankle sprain motion and common sporting motion by ankle inversion velocity. Journal of Biomechanics. 2010;43(10):2035-2038.

111. Saber-Sheikh K, Bryant EC, Glazzard C, et al. Feasibility of using inertial sensors to assess human movement. Man Ther. 2010;15(1):122-125.

112. Tagliamonte NL, Sergi F, Carpino G, et al. Human-robot interaction tests on a novel robot for gait assistance. Paper presented at the Rehabilitation Robotics (ICORR). 2013

113. Belforte G, Gastaldi L, Sorli M. Pneumatic active gait orthosis Mechatronics. 2001;11(3):301-323.

114. Sasaki D, Noritsugu T, Takaiwa M. Development of pneumatic lower limb power assist wear driven with wearable air supply system. Paper presented at the Intelligent Robots and Systems, 2013 IEEE/RSJ International Conference. 2013.
115. Asbeck AT, Dyer RJ, Larusson AF, et al. Biologically-inspired soft exosuit. Paper presented at the Rehabilitation robotics. 2013

116. Kwon S, Park HS, Stanley CJ, et al. A practical strategy for sEMG-based knee joint moment estimation during gait and its validation in individuals with cerebral palsy. IEEE Trans Biomed Eng. 2012;59(5):1480-1487.

117. Li DY, Becker A, Shorter KA, Bretl T, et al. Estimating system state during human walking with a powered ankle-foot orthosis. Mechatronics, IEEE/ ASME Transactions on. 2011;16(5):835-844.

118. Oskoei MA, Hu H. Support vector machine--based classification scheme for myoelectric control applied to upper limb. IEEE Trans Biomed Eng. 2008;55(8):1956-1965.

119. Carrozza MC, Persichetti A, Laschi C, et al. A wearable biomechatronic interface for controlling robots with voluntary foot movements. Mechatronics, IEEE/ASME Transactions. 2007;12(1):1-11.

120. Racine JLC. Control of a lower extremity exoskeleton for human performance amplification. 2003.

121. Guo Q, Jiang D. Method for Walking Gait Identification in a Lower Extremity Exoskeleton based on C4. 5 Decision Tree Algorithm. Int J Adv Robot Syst. 2015;12:30.

122. Desailly E, Daniel Y, Sardain P, et al. Foot contact event detection using inematic data in cerebral palsy children and normal adults gait. Gait Posture. 2009;29(1):76-80.

123. Miller A. Gait event detection using a multilayer neural network. Gait Posture. 2009;29(4):542-545.

124. Rueterbories J, Spaich EG, Larsen B, et al. Methods for gait event detection and analysis in ambulatory systems. Med Eng Phys. 2010;32(6):545-552.

125. Lee JK, Park EJ. Quasi real-time gait event detection using shankattached gyroscopes. Med Biol Eng Comput. 2011;49(6):707-712.

126. Sant'anna A, Wickström N. A symbol-based approach to gait analysis from acceleration signals: Identification and detection of gait events and a new measure of gait symmetry. IEEE Trans Inf Technol Biomed. 2010;14(5):1180-1187.

127. Aung MS, Thies SB, Kenney LP, et al. Automated detection of instantaneous gait events using time frequency analysis and manifold embedding. Neural Systems and Rehabilitation Engineering, IEEE Transactions on. 2013;21(6):908-916.

128. Mansfield A, Lyons GM. The use of accelerometry to detect heel contact events for use as a sensor in FES assisted walking. Med Eng Phys. 2003;25(10):879-885.

129. Gouwanda D, Senanayake S, Arosha M. Application of hybrid multiresolution wavelet decomposition method in detecting human walking gait events. Paper presented at the Soft Computing and Pattern Recognition, SOCPAR'09. 2009.

130. Forsman PM, Toppila EM, Haeggstrom EO. Wavelet analysis to detect gait events. Conf Proc IEEE Eng Med Biol Soc. 2009. p. 424-427.

131. Dejnabadi H, Jolles BM, Casanova E, et al. Estimation and visualization of sagittal kinematics of lower limbs orientation using body-fixed sensors. IEEE Trans Biomed Eng. 2006;53(7):1385-1393.

132. Favre J, Luthi F, Jolles BM, et al. A new ambulatory system for comparative evaluation of the three-dimensional knee kinematics, applied to anterior cruciate ligament injuries. Knee Surg Sports Traumatol Arthrosc. 2006;14(7):592-604.

133. Hidler J. Robotic-assessment of walking in individuals with gait disorders. Conf Proc IEEE Eng Med Biol Soc. 2004;7:4829-4831. 
134. Lee RY. Kinematics of rotational mobilisation of the lumbar spine Clinical Biomechanics. 2001;16(6):481-488.

135. Roduit R, Besse PA, Micallef JP. Flexible angular sensor [and biomechanical application]. Instrumentation and Measurement, IEEE Transactions. 1998;47(4):1020-1022.

136. Sankai Y. Leading edge of cybernics: Robot suit hal. Paper presented at the SICE-ICASE. International Joint Conference. 2006.

137. Santic A, Bilas V, Lackovik I. A system for force measurements in feet and crutches during normal and pathological gait. Periodicum biologorum. 2002;104(3):305-310.
138. Williamson R, Andrews BJ. Gait event detection for FES using accelerometers and supervised machine learning. IEEE Trans Rehabil Eng. 2000;8(3):312-319.

139. Mills PM, Barrett RS, Morrison S. Agreement between footswitch and ground reaction force techniques for identifying gait events: intersession repeatability and the effect of walking speed. Gait Posture. 2007;26(2):323-326. 